\title{
The CDK1 inhibitor RO3306 improves the response of BRCA-proficient breast cancer cells to PARP inhibition
}

\author{
QING XIA ${ }^{1,2^{*}}$, YUCHEN CAI $^{1 *}$, ROUJUN PENG $^{1}$, GUOSHENG WU $^{3}$, YANXIA SHI ${ }^{1}$ and WENQI JIANG \\ ${ }^{1}$ Sun Yat-sen University Cancer Center, State Key Laboratory of Oncology in South China, \\ Collaborative Innovation Center for Cancer Medicine, Guangzhou 510060; ${ }^{2}$ Department of Oncology, \\ Shanghai Jiaotong University Affiliated First People's Hospital, Shanghai 201620; ${ }^{3}$ State Key Laboratory of \\ Quality Research in Chinese Medicine, University of Macau, Macau SAR, P.R. China
}

Received October 9, 2013; Accepted November 13, 2013

DOI: 10.3892/ijo.2013.2240

\begin{abstract}
Breast cancer is one of the most common malignancies in women. Approximately $15 \%$ of the patients belong to the triple-negative breast cancer (TNBC) group, and have the disadvantage of not benefiting from currently available receptor-targeted systemic therapies. Some cancers in the TNBC group harbor defects in DNA double-strand break repair by homologous recombination (HR), such as BRCA1 dysfunction, and are hypersensitive to poly (ADP-ribose) polymerase (PARP) inhibition. However, only a small fraction of the tumors are BRCA-deficient, and this restricts the therapeutic utility of the PARP inhibitor monotherapy. Cyclin-dependent kinase 1 (CDK1) is necessary not only for BRCA1-mediated S phase checkpoint activation, but also for HR, because it phosphorylates BRCA1 for the efficient formation of BRCA1 foci. In this study, we showed that the combined inhibition of CDK1 and PARP in BRCA-proficient MDA-MB-231 breast cancer cells resulted in dramatically reduced cell growth compared to PARP inhibition alone. Mechanistic investigations revealed that this sensitivity appears to be mediated by sustained DNA damage and inefficient DNA repair triggering mitochondrial-mediated apoptosis as well as autophagy. Our results suggest
\end{abstract}

Correspondence to: Professor Wenqi Jiang or Professor Yanxia Shi, Sun Yat-sen University Cancer Center, 651 Dongfeng East Road, Guangzhou 510060, P.R. China

E-mail: jiangwenqi2009@126.com

E-mail: shiyanxia2007@126.com

${ }^{*}$ Contributed equally

Abbreviations: TNBC, triple-negative breast cancer; DSB, doublestrand break; HR, homologous recombination; NER, nucleotide excision repair; BER, base excision repair; PARP, poly (ADP-ribose) polymerase; CDK1, cyclin-dependent kinase 1; GI50, 50\% growth inhibition; CI, combination index; PI, propidium iodide

Key words: cyclin-dependent kinase 1, BRCA1, poly (ADP-ribose) polymerase inhibitor, breast cancer that CDK1 inhibition represents a plausible strategy for expanding the utility of PARP inhibitors to BRCA-proficient breast cancers.

\section{Introduction}

Breast cancer is one of the most common malignancies in women. Approximately $15 \%$ of breast cancer cases belong to the triple-negative breast cancer (TNBC) group, in which neither estrogen/progesterone receptors nor HER2 expression can be detected (1). Systemic treatment for patients with triple-negative disease is currently limited to chemotherapy, and survival of patients in this group is poor compared to patients with other cancer subtypes. BRCA1 is known to be involved in a number of DNA repair pathways, including DNA double-strand break (DSB) repair through homologous recombination $(\mathrm{HR})(2,3)$, nucleotide excision repair (NER) (4) and base excision repair (BER) (5). Some TNBCs harbor defects in DNA double-strand break repair by $\mathrm{HR}$, such as BRCA1 dysfunction, and are hypersensitive to the inhibition of poly (ADP-ribose) polymerase (PARP) (6-9). However, BRCA-mutant tumors represent only $2-3 \%$ of all breast cancers (10) and only $12.5 \%$ of TNBCs (1), which might restrict the therapeutic utility of PARP inhibitor monotherapy. Cyclin-dependent kinase 1 (CDK1) is a master modulator of the initiation and transition process through mammalian mitosis $(11,12)$. Previous studies have shown that CDK1 activity loss or its aberrant expression are involved in the $\mathrm{G} 2 / \mathrm{M}$ phase arrest in many tumor types (13), and that CDK1 inhibition downregulates survival and induces apoptosis $(14,15)$. Besides, CDK1 phosphorylates BRCA1, and this is necessary for its ability to efficiently form BRCA1 foci at DNA damage sites and facilitate checkpoint activation (16). Therefore, CDK1 is considered as an important therapeutic target (15). It is likely that the reduced CDK1 activity may also sensitize BRCA-proficient tumor cells to PARP inhibition, facilitating the extension of the synthetic lethal therapeutic option to a larger patient population. Here we show that the cytotoxic effect of CDK1 and PARP inhibition, administered in a sequential combination regimen, was superior over PARP inhibition alone in the MDA-MB-231 BRCA-proficient breast cancer cell line. 
Combined inhibition resulted in sustained DNA damage, and this was paralleled by a dramatic G2/M cell cycle arrest and the induction of cell death. CDK1 inhibition represents a plausible strategy for expanding the utility of PARP inhibitors to BRCA-proficient breast cancers.

\section{Materials and methods}

Cell lines and drugs. The MDA-MB-231, HCC1937, SK-BR-3, and MCF-7 human breast cancer cell lines were obtained from the Cell Bank of the Chinese Academy of Sciences (Shanghai, China). MDA-MB-231, SK-BR-3 and MCF-7 cells were grown in DMEM media (Invitrogen, Carlsbad, CA, USA) with $10 \%$ fetal bovine serum (FBS) (HyClone, Logan, UT, USA), and HCC1937 cells were cultured in RPMI-1640 (Invitrogen) supplemented with $10 \%$ FBS. All cells were cultured in a $5 \% \mathrm{CO}_{2}$ incubator at $37^{\circ} \mathrm{C}$.

AZD2281 (olaparib) was purchased from Selleck Chemicals (Houston, TX, USA) and $100 \mathrm{mM}$ stocks were prepared. RO3306 was purchased from EMD Chemicals (Gibbstown, NJ, USA) and diluted to a $10-\mathrm{mM}$ stock solution. All stock solutions were prepared using dimethyl sulfoxide (DMSO) as a solvent and stored at $-20^{\circ} \mathrm{C}$.

Analysis of BRCA1 mutations. Genomic DNA was extracted from HCC1937 and MDA-MB-231 using the TIANamp Genomic DNA Kit (Tiangen, Beijing, China). The complete coding regions and the BRCAl exon-intron boundaries were screened by a polymerase chain reaction (PCR)-sequencing assay. The whole BRCAl coding sequence was amplified with 31 pairs of primers. Amplification of DNA fragments was performed in a thermocycler (Gene Cycler ${ }^{\mathrm{TM}}$, Bio-Rad, Hercules, CA, USA) in $25 \mu 1$ reactions containing $30 \mathrm{ng}$ of genomic DNA, $2.5 \mathrm{mM}$ dNTP, $50 \mathrm{mM} \mathrm{MgCl}{ }_{2}, 10 \mathrm{X}$ PCR buffer, $0.5 \mu \mathrm{M}$ of each primer, and 1.25 units of AmpliTaq DNA polymerase (Promega, Madison, WI, USA). The reactions were initially kept at $94^{\circ} \mathrm{C}$ for $3 \mathrm{~min}$ to activate the Taq DNA polymerase, followed by $30 \mathrm{sec}$ denaturation at $94^{\circ} \mathrm{C}, 30 \mathrm{sec}$ annealing at a temperature suitable for each primer pair, and $30 \mathrm{sec}$ extension at $72^{\circ} \mathrm{C}$. The PCR was run for 35 cycles and a 10 min elongation step was performed at the end. All fragments were sequenced using the BigDye Terminator Cycle Sequencing Kit and an ABI 3730XL automated sequencer (Applied Biosystems, Foster City, CA, USA). Each mutation was confirmed in duplicate.

MTT assay and combination effect. Log phase cells $(25,000)$ were seeded in 96 -well plates and incubated in a $37^{\circ} \mathrm{C}$ incubator with $5 \% \mathrm{CO}_{2}$. After $24 \mathrm{~h}$, different concentrations of the PARP inhibitor AZD2281 in the case of the four cell lines, or of the CDK1 inhibitor RO3306 as a single agent in the case of MDA-MB-231, were administered to determine the drug concentrations required to achieve a 50\% growth inhibition (GI50). For combination treatments, MDA-MB-231 cells were treated with AZD2281 or with the sequential combination regimen (RO3306 alone for $4 \mathrm{~h}$ followed by AZD2281 for an additional 72-h period) at the indicated doses. MTT ( $20 \mu \mathrm{l} ; 5 \mathrm{mg} / \mathrm{ml}$ stock solution in saline) was added to each well and the cells were incubated for $4 \mathrm{~h}$. Supernatants were removed and formazan crystals from viable cells were solu- bilized with $200 \mu \mathrm{l}$ anhydrous DMSO. The absorbance was detected with a 550 model microplate reader at the $565 \mathrm{~nm}$ wavelength (17024, Bio-Rad).

The combination effect was evaluated by the combination index (CI), which was calculated using the Calcusyn software (Biosoft, Cambridge, UK). The definition of CI is as follows: $\mathrm{CI}=(\mathrm{D})_{1} /(\mathrm{Dx})_{1}+(\mathrm{D})_{2} /(\mathrm{Dx})_{2}+(\mathrm{D})_{1}(\mathrm{D})_{2} /(\mathrm{Dx})_{1}(\mathrm{Dx})_{2}$, where $(\mathrm{Dx})_{1}$ and $(\mathrm{Dx})_{2}$ are the concentrations of the individual drugs required to produce an $\mathrm{X} \%$ effect alone, and $(\mathrm{D})_{1}$ and $(\mathrm{D})_{2}$ are the concentrations of the combination required to produce the same $\mathrm{X} \%$ effect. The combination effects were defined as follows: $\mathrm{CI}<1$, synergistic effect; $\mathrm{CI}=1$, additive effect; and $\mathrm{CI}>1$, antagonistic effect.

RNA interference. MDA-MB-231 cells were transiently transfected with $50 \mathrm{nM}$ BRCA1 or CDK1 siRNA [5'-GCA GUG AAG AGA UAA AGA ATT-3' (\#1304, Shanghai GenePharma Co., Ltd, Shanghai, China) and 5'-GGG GUU CCU AGU ACU GCA A dTdT-3' (\#2012, Ribo Bio Co., Ltd, Guangzhou, China), respectively], or with a non-targeting control [5'-UUC UCC GAA CGU GUC ACG UTT-3' (\#0420 Shanghai GenePharma Co., Ltd); and 5'-GUU CGC GUU ACG CGA GAU A dTdT-3' (\#7021, Ribo Bio Co., Ltd), respectively], using the TurboFect siRNA transfection reagent (Fermentas Life Sciences, Pittsburgh, PA, USA) in accordance to the manufacturer's instructions. Following a 6-h incubation period, fresh AZD2281-containing growth medium was added and the MTT assay was performed after an additional 72-h incubation period. For western blot analysis, cells were harvested $48 \mathrm{~h}$ post-transfection.

Cell cycle and apoptosis analysis. MDA-MB-231 cells $\left(10 \times 10^{4}\right.$ cells $\left./ \mathrm{ml}\right)$ were treated in 6 -well plates with $50 \mu \mathrm{M}$ AZD2281, $5 \mu \mathrm{M}$ RO3306, or the sequential combination regimen $(5 \mu \mathrm{M}$ RO3306 alone for $4 \mathrm{~h}$ followed by $50 \mu \mathrm{M}$ AZD2281) for $48 \mathrm{~h}$, as described previously. For cell cycle analysis, cells were fixed in 70\% ice-cold ethanol and stained with a propidium iodide (PI) solution $(25 \mu \mathrm{g} / \mathrm{ml}$ PI, $180 \mathrm{U} / \mathrm{ml}$ RNase and $0.1 \%$ Triton X-100). For apoptosis analysis, staining was then performed using the Annexin V-fluorescein isothiocyanate apoptosis detection kit (BestBio, Shanghai, China) according to the manufacturer's instruction. Both cell cycle distribution and apoptosis were analyzed by flow cytometry (FC500; Beckman-Coulter, Brea, CA, USA) and the results are displayed as histograms.

Cell shape assay. Cells were seeded directly in 6-well culture plates at a density of $2 \times 10^{5}$ per well $24 \mathrm{~h}$ and treated with $50 \mu \mathrm{M}$ AZD2281, $5 \mu \mathrm{M}$ RO3306, or the sequential combination regimen $(5 \mu \mathrm{M}$ RO3306 alone for $4 \mathrm{~h}$ followed by $50 \mu \mathrm{M}$ AZD2281) for $48 \mathrm{~h}$, as described previously. Cells were then stained with DAPI and mounted to an inverted microscope (Olympus, IX71).

Western blot analysis. Lysates were prepared from $4 \times 10^{5}$ cells by dissolving pellets in $100 \mu \mathrm{l}$ of lysis buffer $(20 \mathrm{mM}$ $\mathrm{Na}_{2} \mathrm{PO}_{4} \mathrm{pH}=7.4,150 \mathrm{mM} \mathrm{NaCl}, 1 \%$ Triton X-100, $1 \%$ aprotinin, $1 \mathrm{mM}$ phenylmethylsulfonyl fluoride, $10 \mathrm{mg} / \mathrm{ml}$ leupeptin, $100 \mathrm{mM} \mathrm{NaF}$ and $2 \mathrm{mM} \mathrm{Na}_{3} \mathrm{VO}_{4}$ ). Lysates were centrifuged at 14,000 rpm for $20 \mathrm{~min}$. The supernatants were 

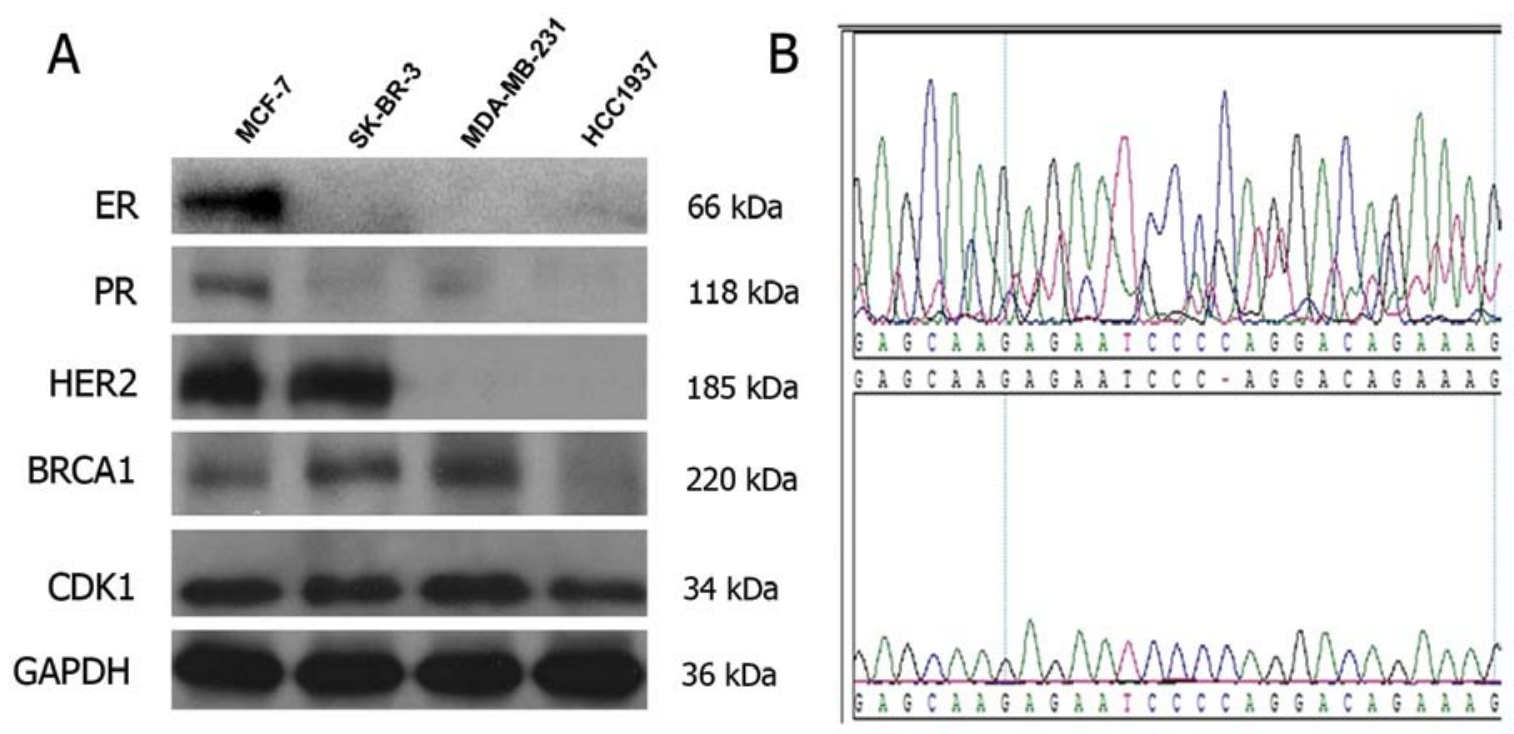

Figure 1. The characteristics of breast cancer cell lines. (A) ER, PR, HER2, BRCA1, CDK1 expression in MCF7, SK-BR-3, MDA-MB-231 and HCC1937 by western blot analysis. (B) Identification of the BRCA1 gene. 19 exon, 73-74insC mutation was detected in HCC1937. Middle, the wild-type sequence. Top and bottom, electrophoretograms displaying the mutation.

collected, and the protein content was determined using the Bio-Rad protein assay. Protein $(30 \mu \mathrm{g})$ was loaded in each well of a 6-15\% SDS-PAGE gel. After resolving the proteins, they were electrophoretically transferred to a nitrocellulose membrane and incubated sequentially with primary antibody and horseradish peroxidase-conjugated goat anti-mouse IgG (zs-2305, Zhongshan Golden Bridge Biotechnology, Beijing, China) or goat anti-rabbit IgG (zs-2301, Zhongshan Golden Bridge Biotechnology). After washing, the bound antibody complex was detected using the LumiGLO reagent (\#7003, Cell Signaling Technology, Danvers, MA, USA) and XAR film (Kodak, XBT-1) as described by the manufacturers. The following primary antibodies were used: ER antibody (\#1115-1, Epitomics, Burlingame, CA, USA), PR antibody (\#5132-1, Epitomics,), HER2 antibody (\#2165, Cell Signaling Technology), BRCA1 antibody (sc-642, Santa Cruz Biotechnology, Santa Cruz, CA, USA), phospho-BRCA1 antibody (\#9009, Cell Signaling Technology), CDK1 antibody (\#9116, Cell Signaling Technology), caspase-3 antibody (\#9662, Cell Signaling Technology), PARP antibody (sc-7150, Santa Cruz Biotechnology), Bcl-2 antibody (\#2870, Cell Signaling Technology), Bax antibody (\#2772, Cell Signaling Technology), caspase-9 antibody (\#9502, Cell Signaling Technology), caspase-8 antibody (\#9746, Cell Signaling Technology), LC3 antibody (NB100-2220, Novus Biologicals, Littleton, CO, USA), P62 antibody (647702, BioLegend, San Diego, CA, USA), beclin 1 antibody (\#3738, Cell Signaling Technology), phospho-AKT (ser473) antibody (\#4058, Cell Signaling Technology), AKT antibody (\#2967, Cell Signaling Technology), phospho-mTOR (ser2448) antibody (\#2971, Cell Signaling Technology), mTOR antibody (\#4517, Cell Signaling Technology), heat shock protein 70 antibody (sc-24, Santa Cruz Biotechnology) and glyceraldehyde 3-phosphate dehydrogenase antibody (KC-5G4, KangChen Bio-tech, Shanghai, China).
Confocal microscopy and indirect immunofluorescence. MDA-MB-231 cells were grown on glass coverslips and treated with $50 \mu \mathrm{M}$ AZD2281, $5 \mu \mathrm{M}$ RO3306, or $5 \mu \mathrm{M}$ RO3306 alone for $4 \mathrm{~h}$ followed by $50 \mu \mathrm{M}$ AZD2281 for $24 \mathrm{~h}$. Cells were subsequently fixed in $4 \%$ paraformaldehyde and stained overnight with primary antibodies against Rad51 (sc-8349, Santa Cruz Biotechnology) or $\gamma$-H2AX (pSer139) (\#NG1904671, Upstate Biotechnology, Lake Placid, NY, USA). Afterwards, cells were washed with phosphate-buffered saline and incubated for $1 \mathrm{~h}$ at room temperature with either Alexa 488 (Invitrogen) or Alexa 555 (Invitrogen) secondary antibodies for Rad51 or $\gamma-\mathrm{H} 2 \mathrm{AX}$, respectively. Cells were fixed with the ProLong gold antifade reagent with 4',6-diamidino-2-phenylindole (Invitrogen) and cured at room temperature for $24 \mathrm{~h}$ before visualizing. The coverslips were viewed with a laser-scanning confocal microscope (Olympus, FV-1000).

Statistical analysis. All experiments were repeated three times. The results of multiple experiments are given as the mean \pm SE. Statistical analysis was performed using the statistical software package SPSS 17.0. p-values were calculated using a one way ANOVA test and a Student's t-test. $\mathrm{p}<0.05$ was considered to indicate a statistically significant difference.

\section{Results}

Cell line subtypes. Initially, we used western blot assays to confirm the characteristics of the four breast cancer cell lines. MCF-7 was identified as the luminal subtype, SK-BR-3 as the HER2 subtype, and MDA-MB-231 and HCC1937 as the triple negative subtypes. All cell lines expressed CDK1 (Fig. 1A). Besides, HCC1937 rather than MDA-MB-231 carried $B R C A 1$ genetic mutations, harboring the 73-74insC exon (Fig. 1B). 

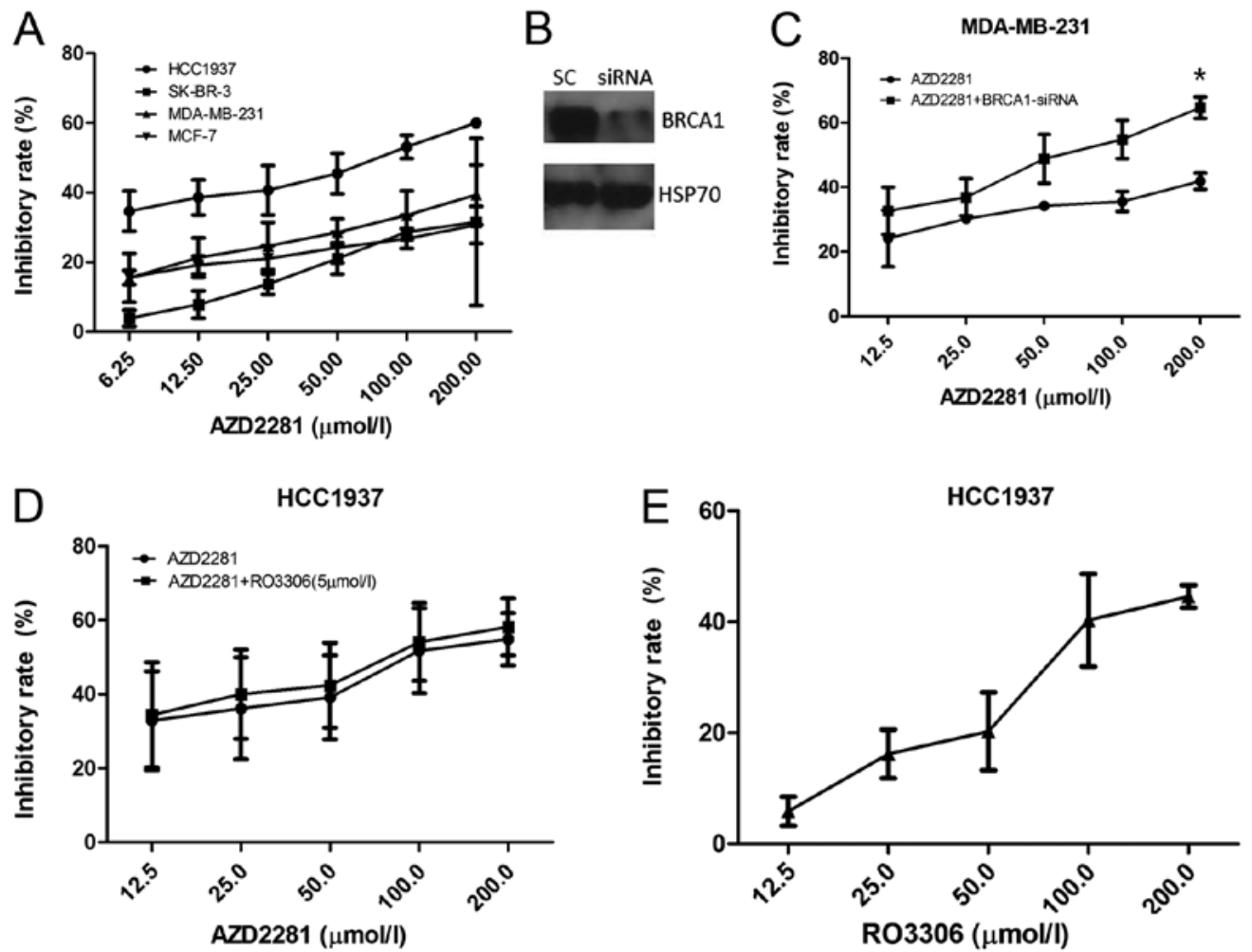

Figure 2. AZD2281 effects on cell viability. (A) Four cell lines were cultured in 96-well plates and AZD2281 was then added for $72 \mathrm{~h}$. (B) MDA-MB-231 cells were transfected with either scrambled (SC) or BRCA1 siRNA. Western blot analysis confirmed BRCA1 knockdown. (C) BRCA1-knockdown MDA-MB-231 cells were treated with AZD2281 for $72 \mathrm{~h}$ (" $\mathrm{p}=0.047$ ). (D and E) HCC1937 cells were exposed to either AZD2281 or RO3306 alone, or to RO3306 for $4 \mathrm{~h}$, followed by exposure to AZD2281 for $72 \mathrm{~h}$ ( $\mathrm{p}=0.547$ ). The MTT assay was used to detect cell viability. The optical density (OD) value of the control was regarded as $100 \%$. Data points are presented as the mean \pm SE.

Sensitivity to PARP inhibition. First, we examined the viability of breast cancer cell lines by the MTT assay in the presence of the PARP inhibitor AZD2281. The BRCAl mutated cell line HCC1937 was the most sensitive, with GI50 of $68.19 \mu \mathrm{M}$ at $72 \mathrm{~h}$, followed by MDA-MB-231, MCF-7 and SK-BR-3 (Fig. 2A). After BRCA1 siRNA transfection, the effects of AZD2281 in MDA-MB-231 were dramatically enhanced ( $p=0.047$, Fig. 2B and C). Furthermore, HCC1937 cells were exposed either to AZD2281 or RO3306 alone or to $5 \mu \mathrm{M}$ RO3306 for $4 \mathrm{~h}$, followed by exposure to AZD2281 for $72 \mathrm{~h}$, and the inhibition rates were nearly the same between AZD2281 and the combination group $(\mathrm{p}=0.547$, Fig. 2D and E), confirming that AZD2281-caused cell growth inhibition depends on the BRCA1 function.

Sensitivity to CDK1 inhibition. In order to examine whether AZD2281 combined with RO3306 was superior over PARP inhibition alone in the BRCA-proficient breast cancer cell line MDA-MB-231, we first determined the effect of RO3306 on reduction in cell counts and the GI50 value of $55.72 \mu \mathrm{M}$ at $48 \mathrm{~h}$ and $7.11 \mu \mathrm{M}$ at $72 \mathrm{~h}$ (Fig. 3A). Western blot analysis to determine the BRCA1 phosphorylation status at Ser1524 was performed following the 48 -h treatment regimen. Upon RO3306 treatment, Ser1524 phosphorylation levels decreased significantly in a dose-dependent manner (Fig. 3B), confirming that the observed effect of RO3306 was associated with inhibition of the targeted BRCA1 phos- phorylation rather than total BRCA1. Rad51 foci represent a crucial component of the HR repair machinery (17) and $\gamma-\mathrm{H} 2 \mathrm{AX}$ is an early response marker for DSB DNA damage signaling (18). We assayed p-BRCA1, Rad51, and $\gamma$-H2AX foci formation by immunofluorescence. After a 24-h treatment with $5 \mu \mathrm{M}$ RO3306, decreased p-BRCA1 and Rad51 foci levels and an increase in $\gamma-\mathrm{H} 2 \mathrm{AX}$ were found (Fig. 3C).

Reduced CDKI activity sensitizes cells to PARP inhibition. We investigated whether CDK1 inhibition could potentiate the growth inhibitory effects of PARP inhibition. First, the CI values of AZD2281: RO3306 = 40:1, 20:1, 10:1, 5:1, 2.5:1, 2:1, 1:1, 1:2, 1:4 and 1:8 were detected. The results showed that the 10:1 ratio exerted the strongest synergistic effect, with the combination index of 0.077 (Fig. 4A). Subsequently, we studied whether significant growth inhibition could be observed upon combining sub-optimal RO3306 doses (doses $\leq$ GI50). When used as a single agent over $72 \mathrm{~h}, 2.5$ and $5 \mu \mathrm{M}$ RO3306 concentrations reduced MDA-MB-231 cell growth by approximately 35 and $43 \%$, respectively (Fig. 3A); hence, these doses were selected. Results showed that the combination reduced GI50 of AZD2281 in a time- and dosedependent manner, combined with $5 \mu \mathrm{M}$ RO3306, the GI50 significantly reduced to $5.25 \mu \mathrm{M}$ for $72 \mathrm{~h}$ (Fig. 4B and C). CDK1 silencing (Fig. 4E) and sequential treatment with AZD2281 resulted in a significant reduction of cell growth as compared to AZD2281 alone ( $p=0.046$, Fig. 4D), which 

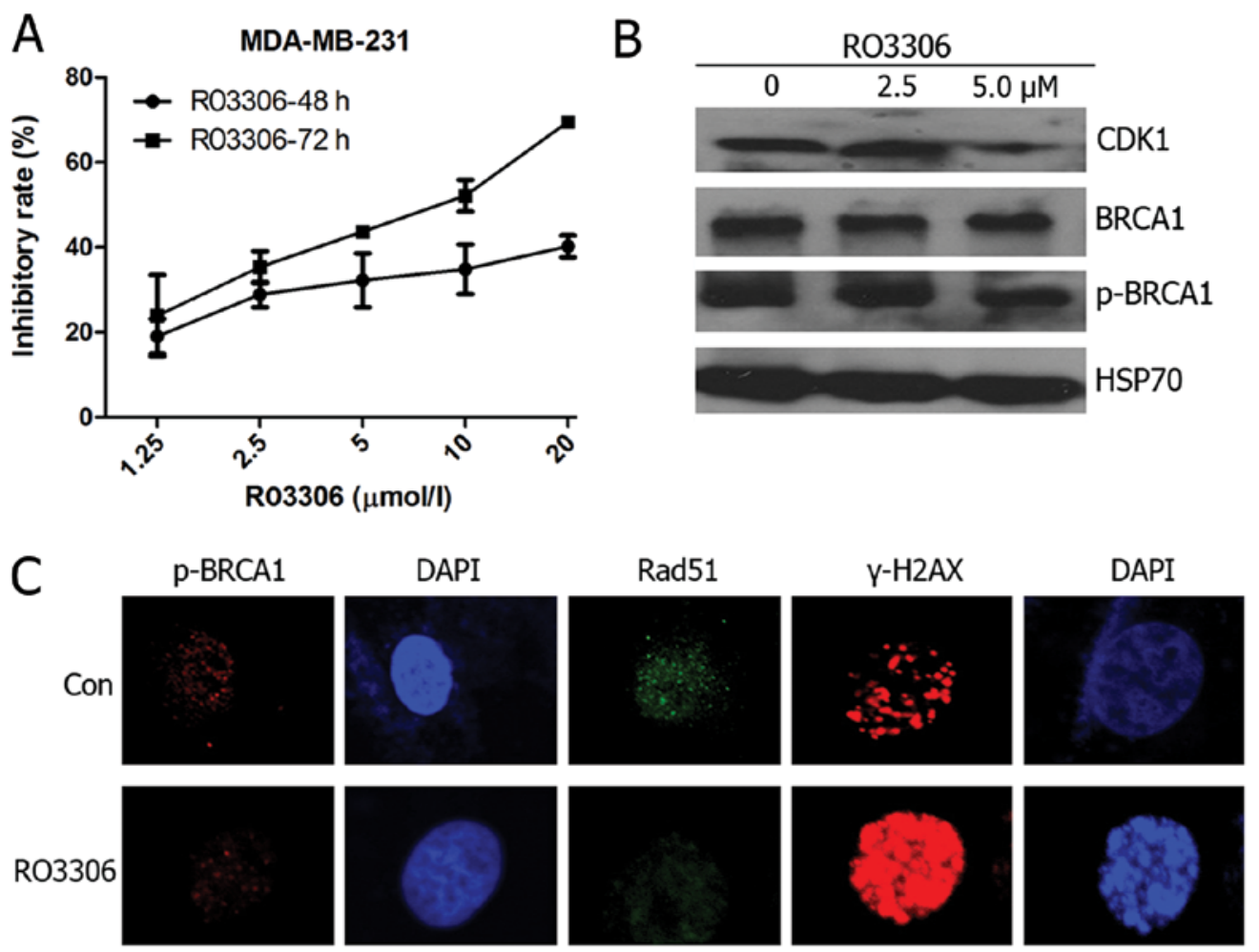

Figure 3. RO3306 effects on MDA-MB-231 viability. (A) Cells were cultured in 96-well plates and RO3306 was then added for 48 or $72 \mathrm{~h}$. The MTT assay was used to detect cell viability. The optical density (OD) value of the control was regarded as $100 \%$. Data points are expressed as the mean \pm SE. (B) MDA-MB-231 cells were exposed to $0.1 \%$ DMSO or RO3306 for $48 \mathrm{~h}$. Representative western blot analysis results are shown for one of three independent experiments. (C) MDA-MB-231 cells were exposed to $0.1 \%$ DMSO or $5 \mu \mathrm{M}$ RO3306 for $24 \mathrm{~h}$. Detection of p-BRCA1, $\gamma$-H2AX, Rad51 and DAPI by immunofluorescence (x1,000).

confirmed the specificity of the CDK1 inhibitors. In addition, if reduced CDK1 activity sensitized cells to PARP inhibition mainly through BRCA1 abrogation, CDK1 depletion should not sensitize BRCA1-deficient cells further. In the absence of CDK1 siRNA, BRCA1 depletion sensitized MDA-MB231 cells to AZD2281 to a similar degree as depletion of CDK1. No further reduction in the inhibition of viability was observed after AZD2281 treatment in cells that were depleted for both BRCA1 and CDK1 (Fig. 4F and G).

Compromising CDK1 and PARP activities induces cell cycle arrest and apoptosis. As shown in Fig. 5A, compared to the control treatment $(57.10 \pm 3.55 \%), 50 \mu \mathrm{M}$ AZD2281, $5 \mu \mathrm{M}$ RO3306, and $5 \mu \mathrm{M}$ RO3306 combined with $50 \mu \mathrm{M}$ AZD2281 reduced the percentage of cells in the G1 phase to $43.20 \pm 1.56 \%, 20.30 \pm 1.95 \%$ and $4.13 \pm 0.68 \%$, respectively $(\mathrm{p}<0.001)$. The percentage of $\mathrm{G} 2 / \mathrm{M}$ cells was $15.93 \pm 3.68 \%$ in the control group and increased to $25.43 \pm 1.71,53.27 \pm 2.21$, and $80.63 \pm 2.25 \%$, respectively $(\mathrm{p}<0.001)$. Furthermore, p-values derived from t-tests comparing AZD2281-treated cells versus cells treated with RO3306 followed by AZD2281 showed a significant percentage of the cells exhibited cell cycle alterations $(\mathrm{p}<0.001)$. These results showed that both AZD2281 and RO3306 induced G2/M phase arrest, and the combination exerted a stronger one. To identify whether CDK1 and PARP inhibition induced apoptosis, treated cells were stained with Annexin V-FITC/PI and the population of apoptotic cells was analyzed by flow cytometry. As seen in Fig. 5B, exposure to $5 \mu \mathrm{M}$ RO3306 followed by $50 \mu \mathrm{M}$
AZD2281 significantly increased the proportion of apoptotic cells. In the control group, $1.80 \pm 1.08 \%$ of the cells were positive for Annexin V-FITC staining, while AZD2281, RO3306 and RO3306 followed by AZD2281 resulted in $12.70 \pm 2.26,20.40 \pm 1.71$ and $29.83 \pm 2.34 \%$, individually $(\mathrm{p}<0.001)$. Furthermore, combination treatments resulted in a significant increase in apoptosis compared to AZD2281 or RO3306 alone ( $<0.05)$. DAPI staining revealed that apoptotic bodies were most typically observed in the combination group (Fig. 5C). To test whether the sequential combination therapy involves increased caspase activation relative to a single agent, we analyzed both the cleavage of the PARP and caspase-3. Western blot analysis demonstrated that although caspase-3 and PARP were modestly cleaved after AZD2281 or RO3306 treatment alone, the levels of cleaved fragments were prominent when both drugs were applied (Fig. 5D). We further tested the expression levels of the proapoptotic protein Bax and the antiapoptotic proteins Bcl-2, results showed that the combination caused a more dramatic Bcl-2 downregulation and Bax upregulation than either drug used alone. These data indicated that both AZD2281 and RO3306 induced apoptosis and that the combination enhanced this effect. Additionally, caspase-9 rather than caspase-8 (data not shown) was cleaved to produce a $37 \mathrm{kDa}$ fragment, indicating that the apoptosis occurred due to a mitochondrial-dependent caspase pathway.

Compromising CDK1 and PARP activities induces autophagy. The growth inhibition rate was about $50 \%$ for the 


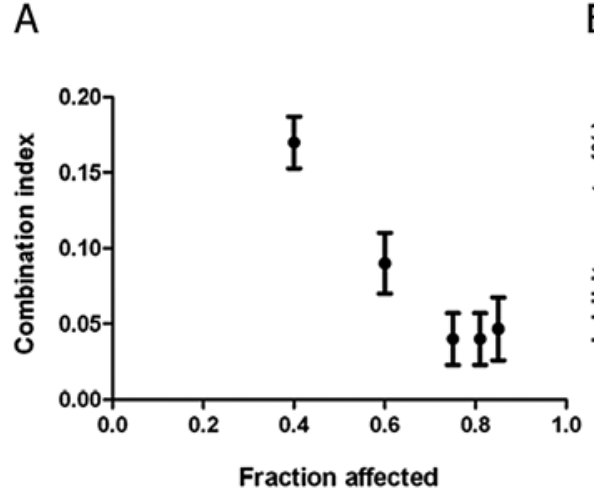

MDA-MB-231

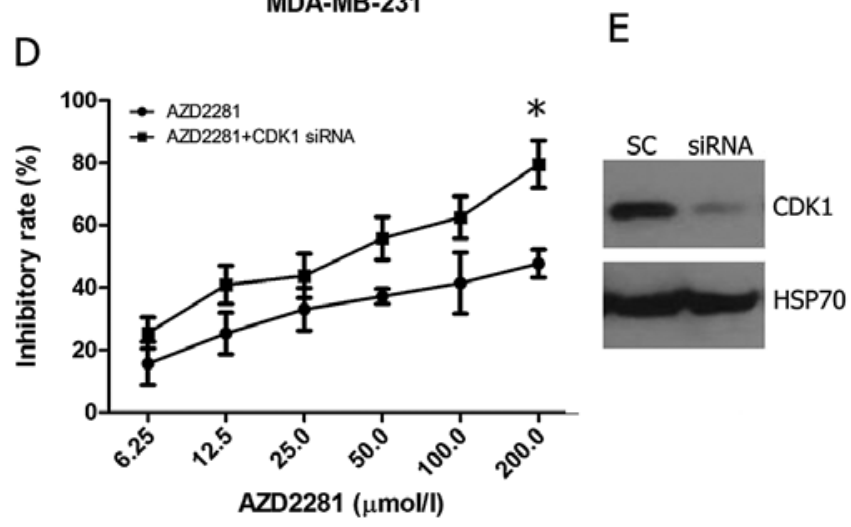

B

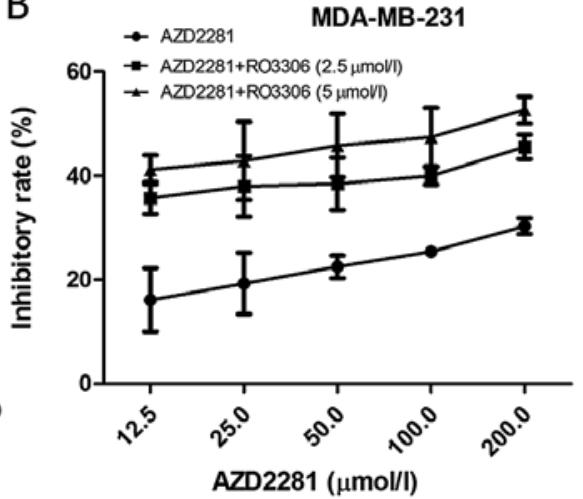

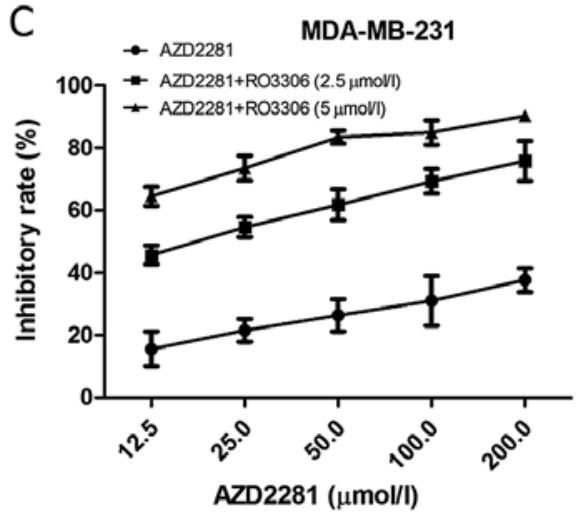

MDA-MB-231

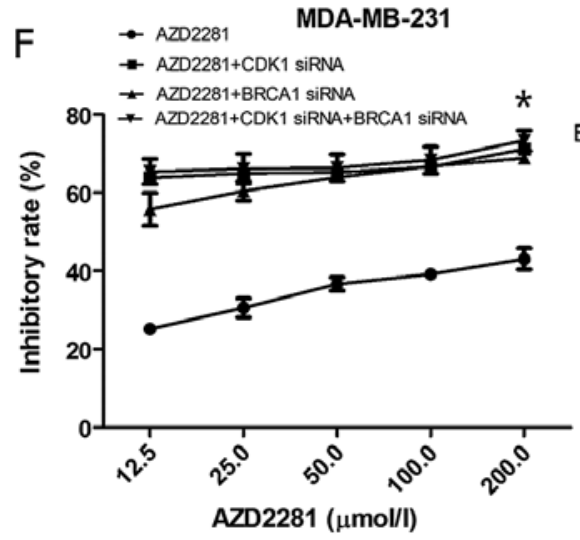

G

BRCA1 SIRNA - +-+

CDK1 SiRNA - + - +

BRCA1

CDK1

HSP70

Figure 4. Effects of drugs on MDA-MB-231 viability. (A) Combination index value of RO3306 followed by exposure to AZD2281 for $72 \mathrm{~h}$ (dose ratio 1:10). (B and C) Cells were exposed to either AZD2281 alone or to RO3306 for $4 \mathrm{~h}$, followed by AZD2281 exposure for (B) 48 or (C) $72 \mathrm{~h}$. (D and E) Cells were transfected with either scrambled (SC) or CDK1 siRNA before treatment with AZD2281 for $72 \mathrm{~h} \mathrm{(} \mathrm{p}=0.046)$. Western blot analysis confirmed CDK1 knockdown. (F and G) Cells were transfected with either scrambled (SC) or BRCA1 siRNA, combined with SC or CDK1 siRNA and then treated with AZD2281 for $72 \mathrm{~h}$. Western blot analysis showed BRCA1 or CDK1 knockdown ( $\mathrm{p}=0.0079$ for control comparing with the other three groups). The MTT assay was used to detect cell viability. The optical density (OD) value of the control was regarded as $100 \%$. Data points are presented as the mean \pm SE.

combined treatment at $48 \mathrm{~h}$ (Fig. 4B), while the apoptosis rate was $29.83 \pm 2.34 \%$ (Fig. 5B). Therefore, it was interesting to test whether other types of cell death were involved. We found that although cells treated with individual drugs showed a very small accumulation of LC3II and beclin 1, cells treated with RO3306 followed by AZD2281 had a strong band indicating an increase in both proteins, and a concomitant P62 reduction (Fig. 5E), showing that not only AZD2281, but also RO3306 induces autophagy and that the combination exacerbates this effect. The exposure to RO3306, followed by AZD2281, inhibited AKT and mTOR phosphorylation, indicating that autophagy was mainly induced by the $\mathrm{PI} 3 \mathrm{~K} / \mathrm{AKT} / \mathrm{mTOR}$ pathway inhibition.

Compromising the CDK1 and PARP activities causes BRCA1 dysfunction and DNA damage. We investigated the effect of AZD2281 and RO3306 on DNA damage by staining for $\operatorname{Rad} 51$ and $\gamma-\mathrm{H} 2 \mathrm{AX}$ to test whether CDK1-inhibited cells would be sensitive to PARP inhibition similarly to BRCA1-deficient cells. As shown in Fig. 6A and B, BRCA1-proficient MDA-MB-231 cells were unable to form Rad51 foci in response to RO3306 followed by AZD2281 treatment, as compared to cells treated with AZD2281 alone $(\mathrm{p}=0.040)$ rather than $\mathrm{RO} 3306(\mathrm{p}=0.663)$, confirming that the homologous recombination defect resulted in unrepaired recombinogenic lesions and cell death. No significant differences existed in the number of $\gamma-\mathrm{H} 2 \mathrm{AX}$ foci between control cells and cells treated with AZD2281, suggesting that AZD2281 alone did not induce DSB. In contrast, the number of $\gamma$-H2AX foci increased dramatically in cells undergoing the sequential combination treatment as compared to AZD2281 $(\mathrm{p}=0.001)$, a finding that was supported by Paull et al (19), who reported that $\gamma$-H2AX foci formed normally in response to DSBs in mammalian cells. We further confirmed the mechanism by showing that BRCA1, p-BRCA1 and CDK1 expression decreased upon RO3306 followed by AZD2281 treatment (Fig. 6C).

\section{Discussion}

The study of the molecular subclasses of breast cancer suggests that treatments should be targeted more selectively to improve outcomes. Currently, a major challenge is to identify such targets and more effective therapeutic regimens for TNBCs. BRCAl is one of the highly penetrant breast cancer susceptibility genes, and the BRCA1 protein fulfills numerous 

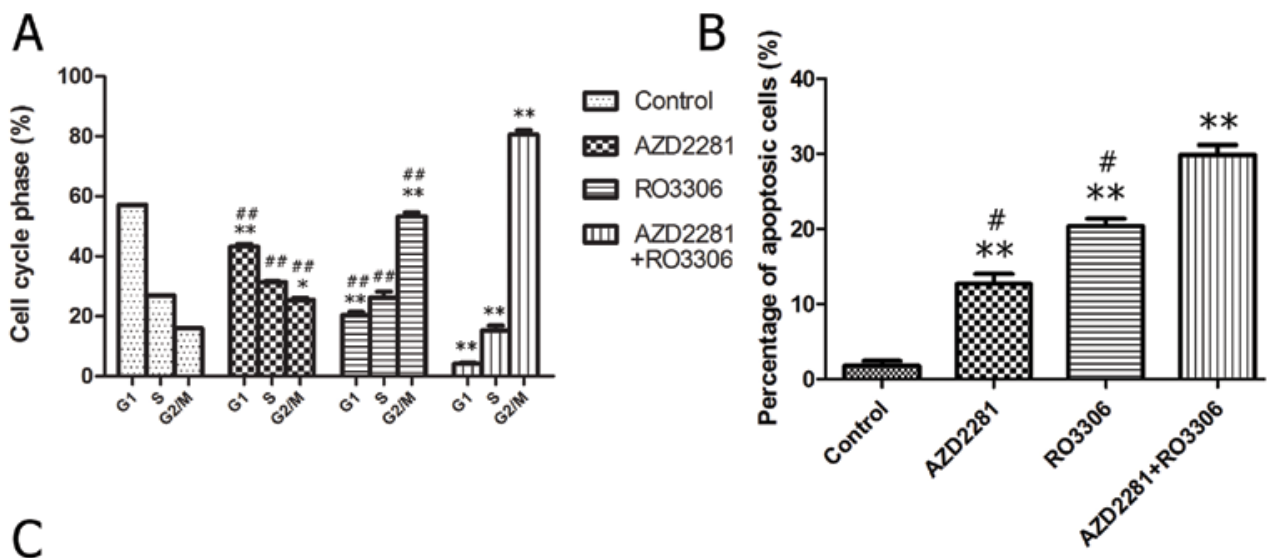

C
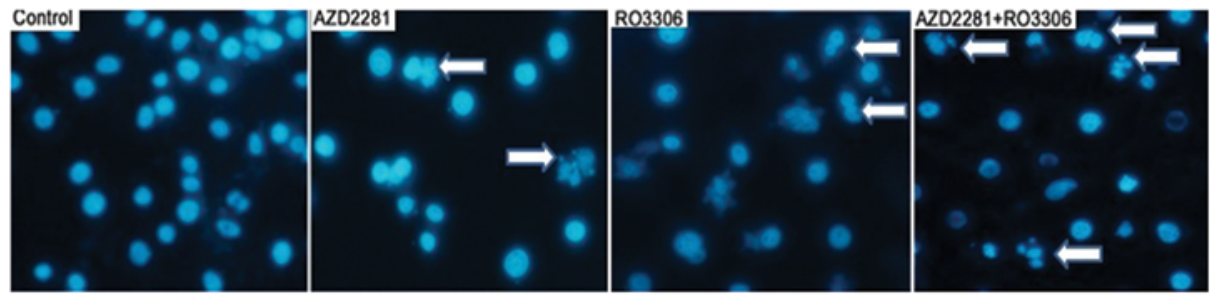

D

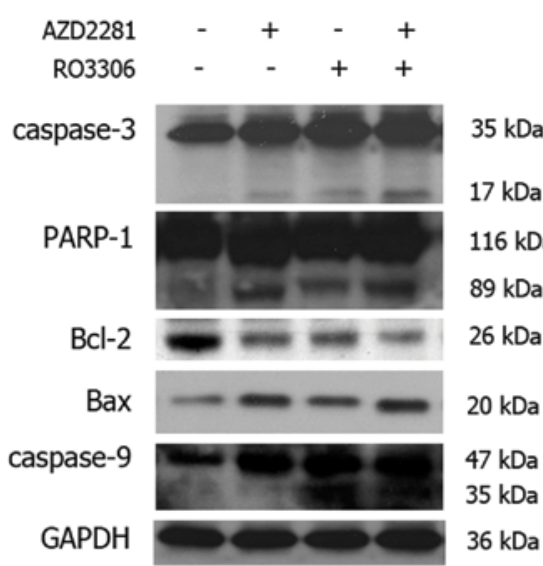

E
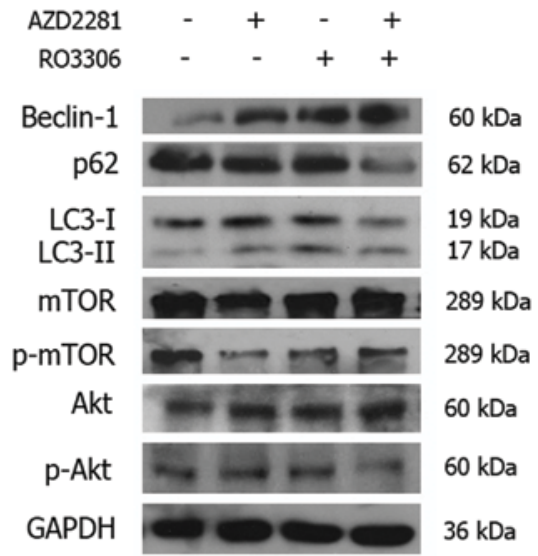

Figure 5. Drugs induced change of cell cycle distribution and apoptosis. Cells were exposed to either $50 \mu \mathrm{M}$ AZD2281 or $5 \mu \mathrm{M}$ RO3306, and for sequential combination studies $4 \mathrm{~h}$ of exposure to $5 \mu \mathrm{M}$ RO3306 were followed by a 48 -h exposure to $50 \mu \mathrm{M}$ AZD2281. (A) Cell cycle distribution results of three independent experiments. (B) Apoptosis results of three independent experiments. [ ${ }^{*} \mathrm{p}<0.05 ;{ }^{* *} \mathrm{p}<0.001$ compared with the control group. ${ }^{*} \mathrm{p}<0.05$; ${ }^{\# \#} \mathrm{p}<0.001$ compared with the combination (AZD2281+RO3306) group]. (C) The morphologic changes of cells were observed by DAPI staining, with apoptotic bodies (arrow) most typically in the combination group. RO3306 cooperated with AZD2281 in (D) inducing mitochondrial apoptosis and (E) autophagy. Representative western blot analysis results are shown from one of three independent experiments.

functions, the best characterized one being related to its role in homologous recombination during DNA repair, chromatin remodeling, DNA decatenation, transcriptional regulation of the estrogen receptor, cell cycle checkpoint control and ubiquitylation (20,21). BRCAl mutations increase the breast cancer risk (22).

PARPs are members of a large family of multifunctional enzymes that play a key role in DNA single-strand break repair through BER (23). In the current study, we established that a BRCA1-deficient rather than a BRCA1-proficient cell line was sensitive to AZD2281. Mechanisms to explain this observation include defects in DNA repair pathways involved in HR. BRCAl germ-line mutation carriers commonly develop DNA repair defects and, therefore, their cells are sensitive to PARP inhibitors (6). However, BRCA1-deficient breast tumors are rare (24), which might restrict the applica- tion of PARP inhibitor monotherapy. Because PARP plays a major role in the BER, we hypothesized that the inhibition of another target, which is indispensable in HR, may act synergistically with PARP inhibitors.

CDK1, a protein that is essential for multiple steps in yeast HR (25), acts as a core component of the cell cycle machinery and forms complexes with cyclins to promote cell cycle progression (12). Genetic ablation of all interphase CDKs (CDK2, CDK4 and CDK6) does not result in cell cycle defects in most cell types, while CDK1 deletion causes cell cycle arrest and prevents embryos from developing beyond the two-cell stage (26). Johnson et al (16) reported that CDK1 is essential for cell division and CDK1 inhibition in lung cancer cells reduces the formation of BRCA1 foci and sensitizes cancer cells to DNA damaging treatments. It is likely that the reduced CDK1 activity may also sensitize cells 


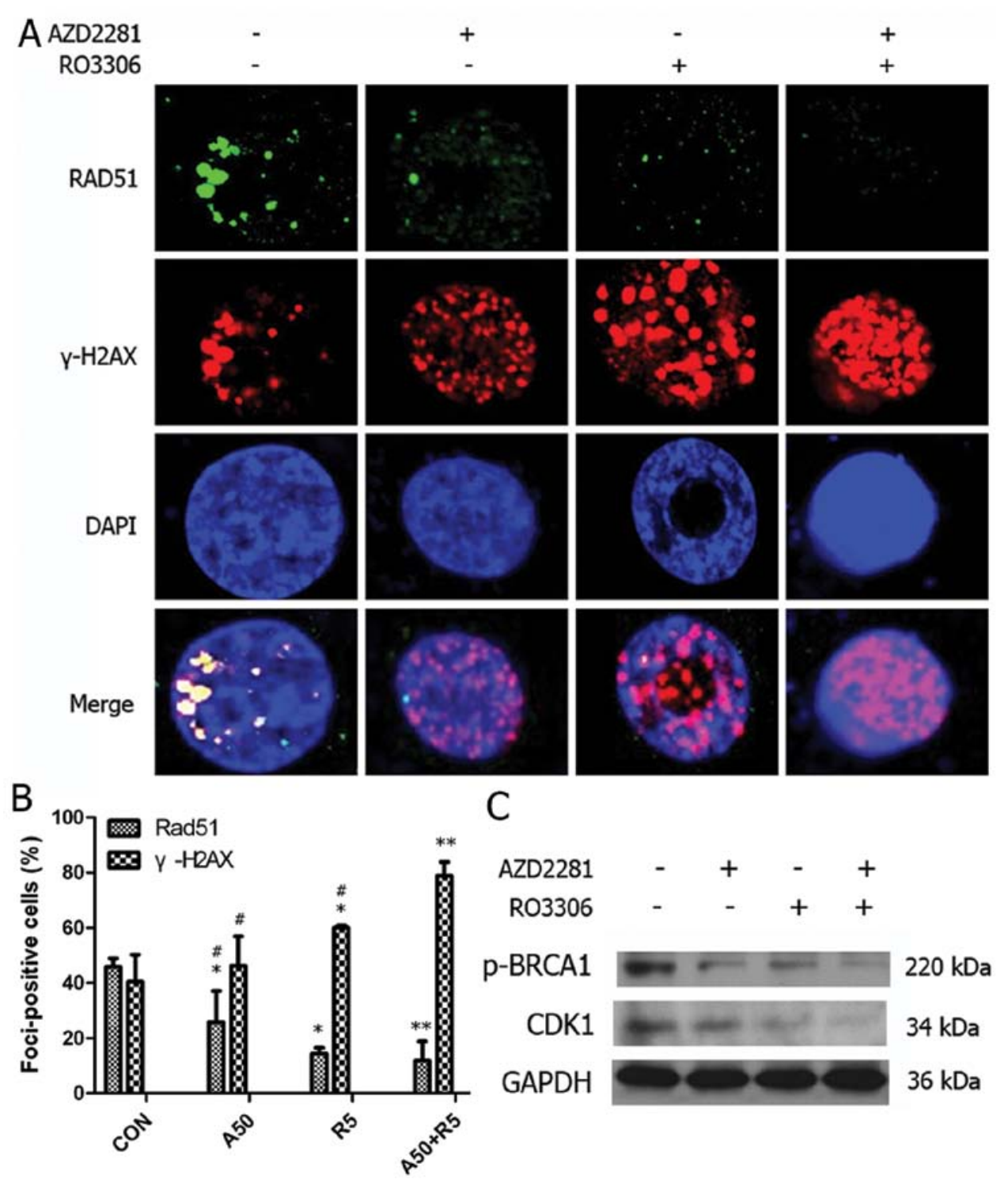

Figure 6. DNA DSB accumulation of MDA-MB-231 after $24 \mathrm{~h}$ of AZD2281 and RO3306 treatment. Cells were exposed to either $50 \mu \mathrm{M}$ AZD2281 or $5 \mu \mathrm{M}$ RO3306 and, for sequential combination studies, $5 \mu \mathrm{M}$ RO3306 for $4 \mathrm{~h}$ followed by $50 \mu \mathrm{M}$ AZD2281. (A) Representative foci-containing cells (x1,000). (B) Number of cells containing $\geq 5 \operatorname{Rad} 51$ and $\gamma$-H2AX foci over three experiments. [" $\mathrm{p}<0.05 ;{ }^{* *} \mathrm{p}<0.001$ compared with the control group. ${ }^{*} \mathrm{p}<0.05$ compared with the combination (AZD2281+RO3306) group]. (C) Detection of p-BRCA1 and CDK1 by western blot analysis. Cells were exposed to either AZD2281 or RO3306 and, for sequential combination studies, to $4 \mathrm{~h}$ of RO3306 followed by a 48-h exposure to AZD2281.

to PARP inhibition by disrupting BRCA1 function in other cancer cells.

We observed that when PARP was inhibited alone, the GI50 of AZD2281 was over $100 \mu \mathrm{M}$ in MDA-MB-231 cells, a finding supported by Lehmann et al (27). However, PARP and CDK1 inhibition together reduced the GI50 dramatically. To our knowledge, studies on the molecular mechanisms of PARP inhibition are limited (28), because most studies focused on characterizing the DNA damage response defects and on measuring PARP activity $(29,30)$. No published reports have shown that PARP inhibition leads to cell death through apoptosis in BRCA-proficient breast cancer cells. To gain more insight into the exact molecular mechanisms involved in decreasing viability following treatment, we investigated several representative apoptosis markers and found that PARP inhibition causes apoptosis. The G2/M phase was associated with
DNA synthesis and the mitotic preparation period, which plays a crucial role in cell cycle progression and the accumulation of cells in the G2/M phase results in cell death. Consistent with Inbar-Rozensal et al (31), who showed that PARP inhibition promotes cell cycle arrest at the $\mathrm{G} 2 / \mathrm{M}$ phase in breast cancer cell lines lacking BRCA1 mutations (MCF-7 and MDA-MB231), we observed that AZD2281 caused G2/M phase arrest. This can be explained by an inhibitory effect that PARP exerts on kinase (ERK)-dependent kinase cascades regulated by extracellular signals, and the resulting decrease in the proportion of putative cancer stem cells (27) and the inhibition of signal transduction pathways involving cell cycle proteins (cyclins, p21, CDK1). On the other hand, by using the CDK1 inhibitor RO3306, we also showed that G2/M phase arrest and apoptosis occur in MDA-MB-231 cells, the finding was supported by Payton et al (32) who found that CDK1 expression was required 
for osteosarcoma and breast tumor cell proliferation and CDK1 suppression decreased the $\mathrm{S}$ phase while markedly increasing the $\mathrm{G} 2 / \mathrm{M}$ phase, thus tumor cells treated with CDK1 inhibitors showed an overall decrease in cell proliferation and apoptosis.

We then investigated the underlying mechanisms by which CDK1 inhibition sensitized cells to PARP inhibition. First, MTT showed that RO3306 followed by AZD2281 treatment inhibited the growth of MDA-MB-231 cells in a time- and dose-dependent manner, with the combination effect synergistic. We subsequently treated MDA-MB-231 cells with the two compounds in sequential combination for flow cytometry and examined not only the dysregulation of the G2/M phase arrest but also the increased proportion of apoptotic cells. The mitochondria-initiated cell death pathway plays an important role in triggering apoptosis in response to stimuli (33) and our data confirmed this. Autophagy is a lysosomal degradation pathway that is essential for survival, development and homeostasis (34). It was previously reported that autophagic cell death has biochemical and morphological features distinguishing it from apoptosis and that some cancer cells could undergo autophagy following cancer therapy (35). We explored both RO3306- and AZD2281-induced autophagy, which were consistent with the prior studies, although the exact mechanism requires further investigation. Furthermore, AZD2281 combined with RO3306 caused dramatic DNA damage refracted by Rad51 and $\gamma-\mathrm{H} 2 \mathrm{AX}$. The experiments above taken together suggested that the cytotoxic effects of the combined inhibition were achieved through the accumulation of DNA DSBs, thereby blocking cells in G2/M and leading to cell death.

In summary, we have used targeted kinase inhibition to inactivate BRCA1, impair the homologous recombination DNA repair, and selectively improve the BRCA-proficient breast cancer cell line MDA-MB-231 to PARP inhibition. Analysis of CDK1-mediated phosphorylation of BRCA1 suggests that a reduction of the CDK1 activity by small molecule inhibitors improved the response to PARP inhibition in vitro, and serves as a guide to translate this to substantial antitumor activity in vivo. The data add substantially to our understanding of the roles that CDK1 and PARP play, and support the clinical development of the combined inhibition. This approach avoids the use of DNA-damaging chemotherapeutic drugs with cellular toxicity, thereby providing the potential to extend well-tolerated PARP inhibition to treating BRCA-proficient breast cancers in the near future.

\section{Acknowledgements}

This study was supported by the National-Eleventh Five technology major project (grant no. 2008ZX09312-002) and the Research Award Fund for Outstanding Young researchers at the Sun Yat-sen Cancer Center.

\section{References}

1. Gucalp A and Traina TA: Triple-negative breast cancer: adjuvant therapeutic options. Chemother Res Pract 2011: 696208, 2011.

2. Moynahan ME, Chiu JW, Koller BH and Jasin M: Brcal controls homology-directed DNA repair. Mol Cell 4: 511-518, 1999.

3. Zhang $\mathbf{J}$ and Powell SN: The role of the BRCA1 tumor suppressor in DNA double-strand break repair. Mol Cancer Res 3: 531-539, 2005.
4. Hartman AR and Ford JM: BRCA1 induces DNA damage recognition factors and enhances nucleotide excision repair. Nat Genet 32: 180-184, 2002.

5. Alli E, Sharma VB, Sunderesakumar P and Ford JM: Defective repair of oxidative DNA damage in triple-negative breast cancer confers sensitivity to inhibition of poly(ADP-ribose) polymerase. Cancer Res 69: 3589-3596, 2009.

6. McCabe N, Turner NC, Lord CJ, Kluzek K, Bialkowska A, Swift S, Giavara S, O'Connor MJ, Tutt AN, Zdzienicka MZ, Smith GC and Ashworth A: Deficiency in the repair of DNA damage by homologous recombination and sensitivity to poly(ADP-ribose) polymerase inhibition. Cancer Res 66: 8109-8115, 2006.

7. Ashworth A: A synthetic lethal therapeutic approach: poly(ADP) ribose polymerase inhibitors for the treatment of cancers deficient in DNA double-strand break repair. J Clin Oncol 26: 3785-3790, 2008.

8. Tutt A, Robson M, Garber JE, Domchek SM, Audeh MW, Weitzel JN, Friedlander M, Arun B, Loman N, Schmutzler RK, Wardley A, Mitchell G, Earl H, Wickens M and Carmichael J: Oral poly(ADP-ribose) polymerase inhibitor olaparib in patients with BRCA1 or BRCA2 mutations and advanced breast cancer: a proof-of-concept trial. Lancet 376: 235-244, 2010.

9. Fong PC, Boss DS, Yap TA, Tutt A, Wu P, Mergui-Roelvink M, Mortimer P, Swaisland H, Lau A, O'Connor MJ, Ashworth A, Carmichael J, Kaye SB, Schellens JH and de Bono JS: Inhibition of poly(ADP-ribose) polymerase in tumors from BRCA mutation carriers. N Engl J Med 361: 123-134, 2009.

10. Wooster R and Weber BL: Breast and ovarian cancer. N Engl J Med 348: 2339-2347, 2003.

11. Malumbres M and Barbacid M: Cell cycle, CDKs and cancer: a changing paradigm. Nat Rev Cancer 9: 153-166, 2009.

12. Morgan DO: Principles of CDK regulation. Nature 374: 131-134, 1995.

13. Vassilev LT, Tovar C, Chen S, Knezevic D, Zhao X, Sun H, Heimbrook DC and Chen L: Selective small-molecule inhibitor reveals critical mitotic functions of human CDK1. Proc Natl Acad Sci USA 103: 10660-10665, 2006.

14. Goga A, Yang D, Tward AD, Morgan DO and Bishop JM: Inhibition of CDK1 as a potential therapy for tumors over-expressing MYC. Nat Med 13: 820-827, 2007.

15. Kojima K, Shimanuki M, Shikami M, Andreeff $M$ and Nakakuma H: Cyclin-dependent kinase 1 inhibitor RO-3306 enhances p53-mediated Bax activation and mitochondrial apoptosis in AML. Cancer Sci 100: 1128-1136, 2009.

16. Johnson N, Cai D, Kennedy RD, Pathania S, Arora M, Li YC, D'Andrea AD, Parvin JD and Shapiro GI: Cdk1 participates in BRCA1-dependent $S$ phase checkpoint control in response to DNA damage. Mol Cell 35: 327-339, 2009.

17. Johnson N, Li YC, Walton ZE, Cheng KA, Li D, Rodig SJ, Moreau LA, Unitt C, Bronson RT, Thomas HD, Newell DR, D'Andrea AD, Curtin NJ, Wong KK and Shapiro GI: Compromised CDK1 activity sensitizes BRCA-proficient cancers to PARP inhibition. Nat Med 17: 875-882, 2011.

18. Gartner EM, Burger AM and Lorusso PM: Poly(adp-ribose) polymerase inhibitors: a novel drug class with a promising future. Cancer J 16: 83-90, 2010.

19. Paull TT, Rogakou EP, Yamazaki V, Kirchgessner CU, Gellert $\mathrm{M}$ and Bonner WM: A critical role for histone H2AX in recruitment of repair factors to nuclear foci after DNA damage. Curr Biol 10: 886-895, 2000.

20. Turner NC and Reis-Filho JS: Basal-like breast cancer and the BRCA1 phenotype. Oncogene 25: 5846-5853, 2006.

21. Gudmundsdottir K and Ashworth A: The roles of BRCA1 and BRCA 2 and associated proteins in the maintenance of genomic stability. Oncogene 25: 5864-5874, 2006.

22. Miki Y, Swensen J, Shattuck-Eidens D, Futreal PA, Harshman K, Tavtigian S, Liu Q, Cochran C, Bennett LM and Ding W: A strong candidate for the breast and ovarian cancer susceptibility gene BRCA1. Science 266: 66-71, 1994.

23. Amé JC, Spenlehauer C and de Murcia G: The PARP superfamily. Bioessays 26: 882-893, 2004.

24. Turner N, Tutt A and Ashworth A: Hallmarks of 'BRCAness' in sporadic cancers. Nat Rev Cancer 4: 814-819, 2004.

25. Ira G, Pellicioli A, Balijja A, Wang X, Fiorani S, Carotenuto W, Liberi G, Bressan D, Wan L, Hollingsworth NM, Haber JE and Foiani M: DNA end resection, homologous recombination and DNA damage checkpoint activation require CDK1. Nature 431: 1011-1017, 2004. 
26. Santamaría D, Barrière $C$, Cerqueira $A$, Hunt $S$, Tardy $C$, Newton K, Cáceres JF, Dubus P, Malumbres M and Barbacid M: Cdk1 is sufficient to drive the mammalian cell cycle. Nature 448: 811-815, 2007.

27. Lehmann BD, Bauer JA, Chen X, Sanders ME, Chakravarthy AB, Shyr Y and Pietenpol JA: Identification of human triple-negative breast cancer subtypes and preclinical models for selection of targeted therapies. J Clin Invest 121: 2750-2767, 2011

28. Kimbung S, Biskup E, Johansson I, Aaltonen K, OttossonWadlund A, Gruvberger-Saal S, Cunliffe H, Fadeel B, Loman N, Berglund $\mathrm{P}$ and Hedenfalk I: Co-targeting of the PI3K pathway improves the response of BRCA1 deficient breast cancer cells to PARP1 inhibition. Cancer Lett 319: 232-241, 2012.

29. Drew Y, Mulligan EA, Vong WT, Thomas HD, Kahn S, Kyle S, Mukhopadhyay A, Los G, Hostomsky Z, Plummer ER, Edmondson RJ and Curtin NJ: Therapeutic potential of poly(ADP-ribose) polymerase inhibito AG014699 in human cancers with mutated or methylated BRCA1 or BRCA2. J Natl Cancer Inst 103: 334-346, 2011

30. Mendes-Pereira AM, Martin SA, Brough R, McCarthy A, Taylor JR, Kim JS, Waldman T, Lord CJ and Ashworth A: Synthetic lethal targeting of PTEN mutant cells with PARP inhibitors. EMBO Mol Med 1: 315-322, 2009.
31. Inbar-Rozensal D, Castiel A, Visochek L, Castel D, Dantzer F, Izraeli $\mathrm{S}$ and Cohen-Armon M: A selective eradication of human nonhereditary breast cancer cells by phenanthridinederived polyADP-ribose polymerase inhibitors. Breast Cancer Res 11: R78, 2009.

32. Payton M, Chung G, Yakowec P, Wong A, Powers D, Xiong L, Zhang N, Leal J, Bush TL, Santora V, Askew B, Tasker A, Radinsky R, Kendall R and Coats S: Discovery and evaluation of dual CDK1 and CDK2 inhibitors. Cancer Res 66: 4299-4308, 2006.

33. Gao LL, Li FR, Jiao P, Yang MF, Zhou XJ, Si YH, Jiang WJ and Zheng TT: Paris chinensis dioscin induces G2/M cell cycle arrest and apoptosis in human gastric cancer SGC-7901 cells. World J Gastroenterol 17: 4389-4395, 2011.

34. Levine B and Klionsky DJ: Development by self-digestion: molecular mechanisms and biological functions of autophagy. Dev Cell 6: 463-477, 2004.

35. Cuervo AM: Autophagy: in sickness and in health. Trends Cell Biol 14: 70-77, 2004. 